\title{
Multiplex Networks of the Guarantee Market: Evidence from China
}

\author{
Shouwei Li and Shihang Wen \\ School of Economics and Management, Southeast University, Nanjing 211189, China \\ Correspondence should be addressed to Shihang Wen; wenzhanyao@gmail.com
}

Received 9 May 2017; Accepted 11 June 2017; Published 9 July 2017

Academic Editor: Benjamin M. Tabak

Copyright (c) 2017 Shouwei Li and Shihang Wen. This is an open access article distributed under the Creative Commons Attribution License, which permits unrestricted use, distribution, and reproduction in any medium, provided the original work is properly cited.

We investigate a multiplex network of the guarantee market with three layers corresponding to different types of guarantee relationships in China. We find that three single-layer networks all have the scale-free property and are of disassortative nature. A single-layer network is not quite representative of another single-layer network. The result of the betweenness centrality shows that central companies in one layer are not necessarily central in another layer. And the eigenvector centrality has the same result of the betweenness centrality except the top central company.

\section{Introduction}

The global financial crisis of 2007-2009 has shown how interconnected the global financial system is. Financial institutions' interconnections can serve as a channel for systemic risk and have been directly linked to the stability of financial systems. Network analysis has contributed to characterizing, understanding, and modeling financial institutions' interconnections, which is gaining popularity across academics, regulators, and policymakers.

There are a lot of studies on financial networks and systemic risk (see, e.g., Allen and Gale [1]; Nier et al. [2]; May and Arinaminpathy [3]; Gai et al. [4]; Li [5]; Li and He [6]; Georg [7]; Sachs [8]; Sensoy and Tabak [9]; Aymanns and Georg [10]; Acemoglu et al. [11]; Chen et al. [12]; Betz et al. [13]; Li et al. [14]; Li and Sui [15]; González-Avella et al. [16]; Sensoy et al. [17]; Christiano Silva et al. [18-21]; Silva et al. [22].), while financial institutions interact in just one way in most of these studies. In fact, financial institutions interact in many ways. Such a situation is best modeled with a multiplex network, where a multiplex network is made up with several layers, each of them composed of all relations of the same type and modeled with a simple network [23]. Multiplex networks can explicitly incorporate multiple channels of connectivity and constitute the natural environment to describe systems interconnected through different categories of connections [24].
Mapping out the structure of complex systems as a monoplex network could lead to missing relevant information [25]. For example, Poledna et al. [26] find that modeling contagion using each layer independently can lead to an underestimation of systemic risk. Therefore, the multiplex network method is useful in improving our understanding of complex systems by taking such multilayer features into account.

The theory of multiplex networks is in its early stages and has been introduced within the last three years to analyze the structure of financial systems [27]. The study of financial multiplex networks has only appeared recently. Empirical analyses of the financial multiplex networks of Colombia, UK, Mexico, Italy, Europe, and USA are provided by León et al. [28], Langfield et al. [29], Molina-Borboa et al. [30], Bargigli et al. [31], Aldasoro and Alves [32], and Musmeci et al. [33], respectively.

León et al. [28] investigate a multiplex network of Colombian sovereign securities settlements corresponding to the three sovereign securities' trading and registering environments and find that the multiplex network has some features, such as sparse, inhomogeneous, scale-free, ultra-small-world, and clustered. Langfield et al. [29] construct the multiplex network of the UK interbank market and find that the network of interbank exposures exhibits a core-periphery structure and the funding network has less of a core-periphery structure. Molina-Borboa et al. [30] analyze the persistence 
and overlap of relationships between banks in a multiplex network of the Mexican banking system, where the multiplex network includes five layers, namely, collateralized loans between banks, new deposits and loans, securities, outstanding deposits, and loans and derivatives. Bargigli et al. [31] analyze the Italian interbank multiplex network by transaction type and by maturity and find that layers have different topological properties and persistence over time. Aldasoro and Alves [32] adopt data on interbank exposures broken down by both maturity and instrument type to investigate structures of the multiplex network of large European banks and find that the network presents positive correlated multiplexity and a high similarity between layers. Musmeci et al. [33] analyze structural properties of the multiplex network of US stock markets, which includes four layers corresponding, respectively, to linear, nonlinear, tail, and partial correlations among a set of financial time series. They find that some features are unique to the multiplex structure and would not be visible otherwise by the separate analysis of the single-layer networks.

In recent years, China's listed companies widely adopt different forms of guarantee to form a complex guarantee circle, which reduces the difficulty of corporate finance to a certain extent. But this also provides risk contagion channels among listed companies. Generally, listed companies who require guarantees tend to have relatively poor performance and relatively large financial risk. Once a listed company has bankruptcy risk, it may result in risk contagion among listed companies through guarantee connections and even lead to systemic risk. Since 2012, the frequency of China's guarantee circle crisis is increasing, such as, the guarantee circle crisis in Zhejiang, Shanghai, Shandong, and other places.

In this paper, we investigate structures of the multiplex network of the guarantee market in China, which would be conducive to preventing risk contagion in the guarantee market. The study on financial multiplex networks is still preliminary. Existing empirical study on financial multiplex networks only analyzes sovereign securities markets, interbank markets, and stock markets. Therefore, this paper contributes to the literature on financial multiplex networks. Second, we study different segments of the guarantee market by guarantee types, and this would help to understand the economics of guarantee market infrastructures as the collective function of several layers of interaction between financial institutions.

The rest of the paper is organized as follows. Section 2 presents the guarantee multiplex network and the data set. Section 3 presents the results of single-layer networks. Section 4 presents the results of multiplex networks. And the conclusion is drawn in Section 5.

\section{Guarantee Multiplex Network and Data Description}

2.1. Guarantee Multiplex Network. We consider a guarantee market consisting of $N$ listed companies and $m$ different types of guarantee relationships. We can adopt the multiplex network to describe the structure of the guarantee market. A network consisting of a type of relationship can be described by a adjacency matrix. Moreover, the structure of the guarantee market can fully be described by the set of adjacency matrices, which is given as follows:

$$
A=\left\{A^{[1]}, A^{[2]}, \ldots, A^{[m]}\right\},
$$

where $A^{[\theta]}=\left\{a_{i j}^{\theta}\right\}$, with $a_{i j}^{\theta}=1$ if there is the $\theta$ type guarantee relationship between listed company $i$ and listed company $j$; otherwise $a_{i j}^{\theta}=0$.

If we consider the amount of the guarantee, we can fully describe the structure of the guarantee market by the set of weighted adjacency matrices,

$$
W=\left\{W^{[1]}, W^{[2]}, \ldots, W^{[m]}\right\},
$$

where $W^{[\theta]}=\left\{W_{i j}^{\theta}\right\}$ and $W_{i j}^{\theta}$ denotes the amount of the $\theta$ type guarantee. In this paper, we only analyze unweighted multiplex networks of the guarantee market in China.

2.2. Data Description. In this paper, we investigate guarantee relationships of listed companies in China, where the data used stem from the Wind database and the time interval is from 2005 to 2015. The Wind database is a leading integrated service provider of financial data in China. In order to simplify data processing, we regard guarantee relationships of subsidiaries as their parent companies'. Besides, if there are more than one guarantee contract between two companies, we assumed that there is only one edge between these companies.

There are 8 types of guarantee relationships among listed companies, namely, joint liability guarantee (JLG), guaranteed guarantee (GUG), credit guarantee (CRG), counter guarantee (COG), general guarantee (GEG), mutual guarantee (MUG), mortgage guarantee (MOG), and pledge guarantee (PLG). Table 1 shows numbers of companies and guarantee relationships from 2005 to 2015. Although various guarantee relationships all play an important role in corporate finance, there are some differences among them. In fact, from Table 1, we can obtain the numbers of the 8 types of guarantee relationships from 2005 to 2015, where the numbers of JLG, GUG, CRG, COG, GEG, MUG, MOG, and PLG are 26126, $1705,710,106,3402,204,1121$, and 551, respectively. It can be seen that the numbers of JLG, GUG, and GEG are the largest three. Therefore, in this paper, we investigate a multiplex network with three layers corresponding, respectively, to JLG, GUG, and GEG. Due to the different time period of guarantees, in this paper we construct networks based on all data from 2005 to 2015 without special instructions.

\section{Structure Analysis of Single-Layer Networks}

We first investigate topological structures of the three singlelayer networks. Figure 1 shows the distributions of degrees of nodes of the JLG network, the GUG network, and the GEG network. It can be seen that degree distributions of these three networks follow a power law, and corresponding exponents are $3.2200,2.9000$, and 2.8900 , respectively. Therefore, three single-layer networks all have the scale-free property. This result means that there is a very strong heterogeneity of the 
TABLE 1: Numbers of companies and guarantee relationships from 2005 to 2015.

\begin{tabular}{cccccccccc}
\hline Year & Number of companies & JLG & GUG & CRG & COG & GEG & MUG & MOG & PLG \\
\hline 2005 & 1869 & 1522 & 135 & 48 & 8 & 154 & 37 & 32 & 20 \\
2006 & 1772 & 1424 & 104 & 42 & 6 & 199 & 40 & 30 & 6 \\
2007 & 1619 & 1207 & 134 & 66 & 8 & 181 & 43 & 35 \\
2008 & 1508 & 1184 & 123 & 36 & 12 & 120 & 29 & 39 \\
2009 & 1291 & 967 & 120 & 34 & 14 & 119 & 23 & 46 \\
2010 & 2808 & 1834 & 395 & 271 & 25 & 170 & 13 & 88 \\
2011 & 2643 & 1937 & 379 & 176 & 29 & 96 & 19 & 86 \\
2012 & 3661 & 3019 & 83 & 6 & 4 & 494 & 0 & 108 \\
2013 & 4354 & 3618 & 78 & 15 & 0 & 545 & 0 & 155 \\
2014 & 4900 & 4102 & 99 & 13 & 0 & 613 & 0 & 51 \\
2015 & 6150 & 5312 & 55 & 3 & 0 & 711 & 0 & 189 \\
\hline
\end{tabular}
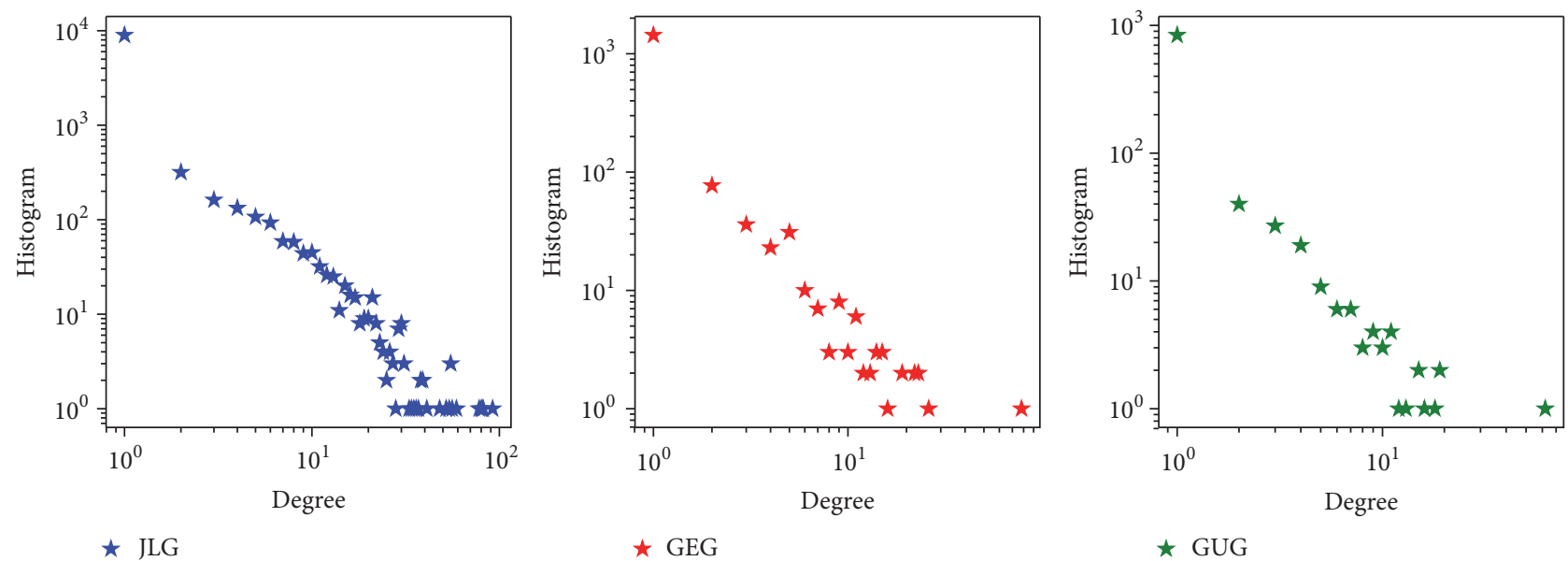

FIGURE 1: Distribution of degrees of nodes of single-layer networks.

single-layer networks, with few important hubs and many nodes with low degree. This means that few listed companies are at the heart of the guarantee market in China. If these listed companies go bankrupt, it is likely to lead to the break of the guarantee chain, which may lead to regional financial crisis.

Next we analyze degree correlations of the three singlelayer networks, which can be measured by the following formula [34]:

$$
=\frac{M^{-1} \sum_{i} j_{i} k_{i}-\left[M^{-1} \sum_{i}(1 / 2)\left(j_{i}+k_{i}\right)\right]^{2}}{M^{-1} \sum_{i}(1 / 2)\left(j_{i}^{2}+k_{i}^{2}\right)-\left[M^{-1} \sum_{i}(1 / 2)\left(j_{i}+k_{i}\right)\right]^{2}},
$$

where $j_{i}, k_{i}$ are the degrees of the nodes at the ends of the $i$ th edge, with $i=1,2, \ldots, M$. This formula measures whether a node of high degree at one end of a link prefers a node of high degree (assortative mixing, $r>0$ ) or low degree (disassortative mixing $r<0$ ) at the other end. According to this formula, we can obtain that $r$ values of JLG, GUG, and GEG networks are $-0.3000,-0.1780$, and -0.1670 , respectively. This indicates that the three single-layer networks are of disassortative nature; that is, companies of high degrees tend to be connected to companies with low degrees. The possible reason for this is that companies tend to find companies with high credit for guarantee, where companies with high credit usually have high degrees. Therefore, it is necessary to identify the so-called systemically important listed companies, which would adversely affect large parts of the guarantee network in case of their bankruptcies.

\section{Structure Analysis of Multiplex Networks}

4.1. Similarity Analysis. In this section, we focus on analyzing the similarity between different layers, where the similarity analysis assesses to what extent a layer is representative of the other. We adopt the Jaccard similarity $J$ to analyze network similarity, where the Jaccard similarity is the probability of observing a link in a network conditional on the observation of the same link in the other network [31]. The Jaccard similarity $J$ is defined as follows [31]:

$$
J(P, Q)=\frac{|P \wedge Q|}{|P \vee Q|}
$$

where $\wedge(\vee)$ is the entry-wise maximum (minimum) of $P$ and Q. 
TABLE 2: Jaccard similarity of the JLG network over time.

\begin{tabular}{|c|c|c|c|c|c|c|c|c|c|c|}
\hline & 2006 & 2007 & 2008 & 2009 & 2010 & 2011 & 2012 & 2013 & 2014 & 2015 \\
\hline 2005 & 0.4430 & 0.3060 & 0.2280 & 0.1710 & 0.0900 & 0.0700 & 0.0500 & 0.0410 & 0.0350 & 0.0270 \\
\hline 2006 & & 0.4590 & 0.3000 & 0.2160 & 0.1140 & 0.0860 & 0.0650 & 0.0470 & 0.0390 & 0.0290 \\
\hline 2007 & & & 0.4660 & 0.3150 & 0.1490 & 0.1090 & 0.0760 & 0.0550 & 0.0440 & 0.0340 \\
\hline 2008 & & & & 0.4560 & 0.2090 & 0.1470 & 0.0950 & 0.0650 & 0.0530 & 0.0390 \\
\hline 2009 & & & & & 0.2730 & 0.1890 & 0.1150 & 0.0780 & 0.0600 & 0.0430 \\
\hline 2010 & & & & & & 0.4120 & 0.2580 & 0.1770 & 0.1410 & 0.1050 \\
\hline 2011 & & & & & & & 0.3390 & 0.2230 & 0.1730 & 0.1260 \\
\hline 2012 & & & & & & & & 0.4640 & 0.3200 & 0.2260 \\
\hline 2013 & & & & & & & & & 0.5030 & 0.3280 \\
\hline 2014 & & & & & & & & & & 0.4840 \\
\hline
\end{tabular}

TABLE 3: Jaccard similarity of the GUG network over time.

\begin{tabular}{lccccccccccc}
\hline & 2006 & 2007 & 2008 & 2009 & 2010 & 2011 & 2012 & 2013 & 2014 & 2015 \\
\hline 2005 & 0.3580 & 0.2510 & 0.1620 & 0.1230 & 0.0470 & 0.0300 & 0.0090 & 0.0090 & 0.0000 & 0.0000 \\
2006 & & 0.4000 & 0.2470 & 0.1850 & 0.0460 & 0.0320 & 0.0330 & 0.0170 & 0.0050 & 0.0060 \\
2007 & & & 0.3110 & 0.2450 & 0.0690 & 0.0410 & 0.0380 & 0.0190 & 0.0040 & 0.0050 \\
2008 & & & & 0.4820 & 0.1090 & 0.0640 & 0.0400 & 0.0260 & 0.0090 & 0.0110 \\
2009 & & & & & 0.1490 & 0.0780 & 0.0410 & 0.0260 & 0.0090 & 0.0120 \\
2010 & & & & & & 0.3460 & 0.0350 & 0.0170 & 0.0100 & 0.0110 \\
2011 & & & & & & & 0.0290 & 0.0130 & 0.0080 & 0.0090 \\
2012 & & & & & & & & & 0.1260 & 0.0580 & 0.0950 \\
2013 & & & & & & & & & & 0.4630 & 0.1570 \\
2014 & & & & & & & & & & & \\
\hline
\end{tabular}

According to (2), we can obtain that the Jaccard similarity $J$ between the JLG network and the GUG network is 0.0340 , and that between the JLG network and the GEG network is 0.0460, and that between the GUG network and the GEG network is 0.0390 . Therefore, the Jaccard similarity between different layers is relatively low, and thus a single-layer network is not quite representative of another single-layer network. This means that the structure varies greatly from one single-layer network to another, due to the difference of guarantee types.

Moreover, we can adopt the Jaccard similarity to analyze the similarity of individual layers over time, where the results are shown in Tables 2-4. From them, we can see that $J$ values of the JLG market are roughly around 0.4500 for two consecutive years, and that of the GUG market and the GEG market have obvious fluctuation. This result shows that guarantee relationships in the JLG market are much more stable than those in the GUG market and the GEG market. Besides, the Jaccard similarity decreases with the increase of the lag phase in the three types of guarantee markets.

Similarity analysis is a relevant tool in assessing the stability of individual layers over time and the similarity between different layers. The above results show that the joint liability guarantee relationship is relatively stable in China's guarantee market and suggest significant complementarity between different segments of the guarantee market.
4.2. Centrality Analysis. Centrality is an important concept of network theory. In the paper we analyze mostly betweenness and eigenvector centrality. The betweenness centrality quantifies how frequently a node acts as a bridge along the shortest path between two other nodes and is computed as follows [23]:

$$
C_{B}(v)=\frac{1}{(n-1)(n-2)} \sum_{i, j \neq v} \frac{N_{i j}(v)}{N_{i j}},
$$

where $C_{B}(v)$ denotes the betweenness of node $v, n$ is the number of nodes in the network, and $N_{i j}$ represents the number of shortest paths between nodes $i$ and $j . N_{i j}(v)$ is the number of shortest paths between nodes $i$ and $j$ that contain node $v$. Figure 2 reports the betweenness centrality in the JLG market either versus the betweenness in the GUG market (Figure 2(a)) or versus the betweenness in the GEG market (Figure 2(b)). From Figure 2, we can know that, in some cases, the betweenness centrality of a company can be markedly different in different layers. Therefore central companies in one layer are not necessarily central in another layer.

Eigenvector centrality is defined as the principal eigenvector of the adjacency matrix defining the network $[35,36]$. If the adjacency matrix of a network is denoted by $A$, then the equation of an eigenvector is as follows:

$$
\lambda \nu=A v,
$$


TABLE 4: Jaccard similarity of the GEG network over time.

\begin{tabular}{|c|c|c|c|c|c|c|c|c|c|c|}
\hline & 2006 & 2007 & 2008 & 2009 & 2010 & 2011 & 2012 & 2013 & 2014 & 2015 \\
\hline 2005 & 0.2700 & 0.1240 & 0.1000 & 0.0920 & 0.0550 & 0.0250 & 0.0170 & 0.0090 & 0.0120 & 0.0060 \\
\hline 2006 & & 0.2030 & 0.1560 & 0.1040 & 0.0540 & 0.0310 & 0.0270 & 0.0120 & 0.0160 & 0.0070 \\
\hline 2007 & & & 0.2920 & 0.1860 & 0.0800 & 0.0340 & 0.0270 & 0.0150 & 0.0180 & 0.0110 \\
\hline 2008 & & & & 0.3740 & 0.1240 & 0.0430 & 0.0230 & 0.0150 & 0.0170 & 0.0120 \\
\hline 2009 & & & & & 0.2040 & 0.1080 & 0.0460 & 0.0260 & 0.0240 & 0.0200 \\
\hline 2010 & & & & & & 0.1320 & 0.0680 & 0.0360 & 0.0340 & 0.0230 \\
\hline 2011 & & & & & & & 0.1030 & 0.0420 & 0.0370 & 0.0240 \\
\hline 2012 & & & & & & & & 0.3550 & 0.2270 & 0.1680 \\
\hline 2013 & & & & & & & & & 0.3820 & 0.2780 \\
\hline 2014 & & & & & & & & & & 0.4490 \\
\hline
\end{tabular}

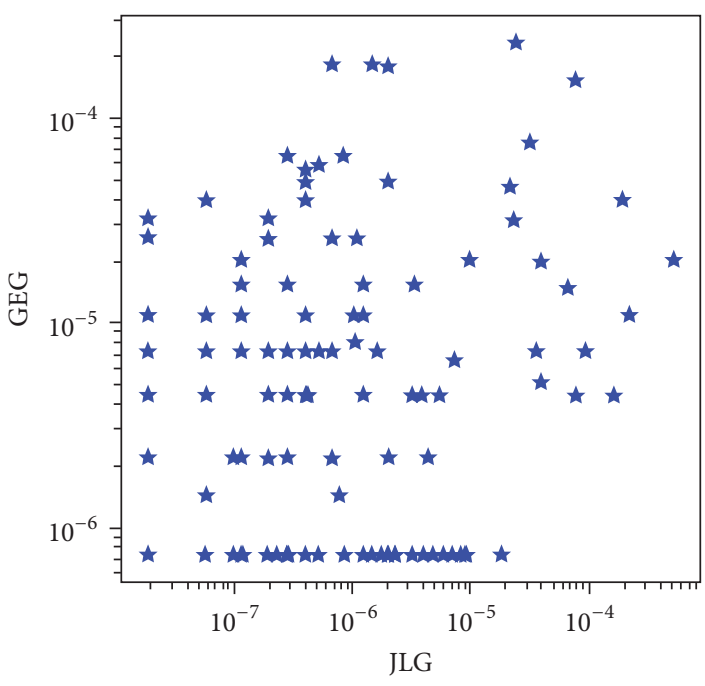

(a)

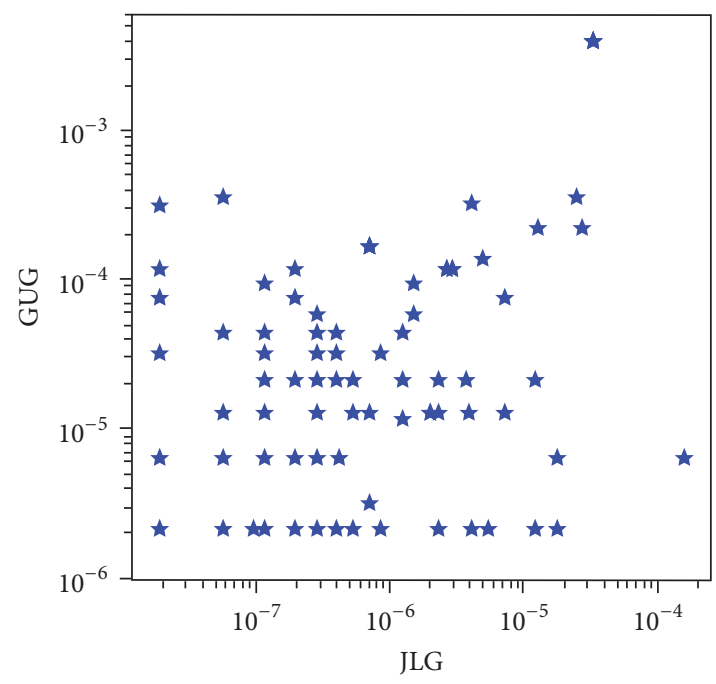

(b)

FIGURE 2: Betweenness centrality of the multiplex network.

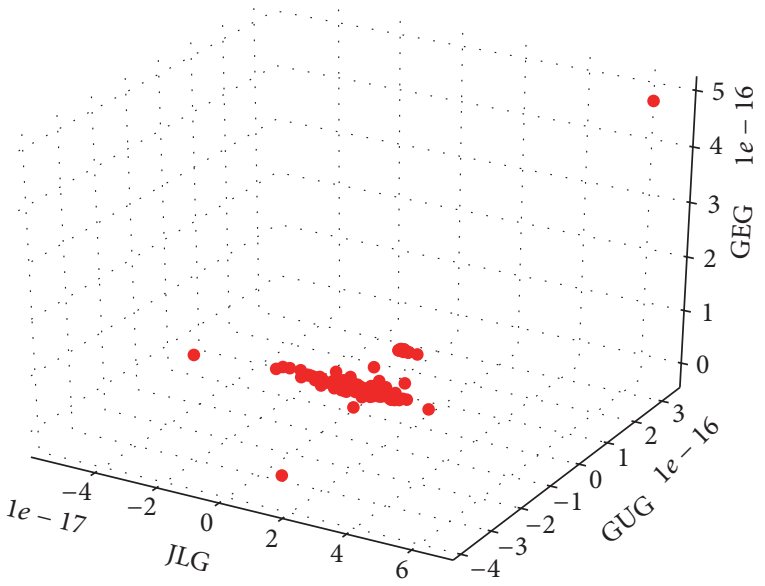

FIGURE 3: Eigenvector centrality of the multiplex network.

where $\lambda$ is a constant and $\nu$ is the eigenvector. Figure 3 shows the three-dimensional plot of the eigenvector centrality of the multiplex network. From it, we can see that the top central node in one layer is also the top central node in another layer. For nodes except the top central node, we can also obtain that the eigenvector centrality of a company can be markedly different in different layers. This result is the same as that of the betweenness centrality.

According to the above analysis, we can see that the centrality measures are important tools, because they can give insight on the degree of specialization of some listed companies as guarantors for some type of guarantee. Therefore, in order to maintain the stability of the guarantee market in China, the centrality measure provides an important tool.

\section{Conclusion}

Financial markets are complex systems, which can be understood better based on network theory. Usually, there are more than one type of relationships between financial institutions. Therefore it is very important to understand financial markets from the perspective of multiplex networks rather than single-layer networks. In this paper, we examine how listed companies relate to each other in different types of guarantee relationships. We investigate a multiplex network with three 
layers corresponding, respectively, to joint liability guarantee, guaranteed guarantee, and general guarantee in China.

First, we find that three single-layer networks all have the scale-free property and are of disassortative nature; that is, companies of high degrees tend to be connected to companies with low degrees. Second, according to similarity analysis, we can know that the Jaccard similarity between different layers is relatively low, and thus a single-layer network is not quite representative of another single-layer network. For individual-layer networks, we find that guarantee relationships in the joint liability guarantee market are much more stable than those in the guaranteed guarantee market and the general guarantee market. And the Jaccard similarity decreases with the increase of the lag phase in the three types of guarantee markets.

Finally, we also investigate the betweenness and eigenvector centrality of a company in the network. The result of the betweenness centrality shows that central companies in one layer are not necessarily central in another layer. And the eigenvector centrality has the same result of the betweenness centrality except the top central company.

\section{Conflicts of Interest}

The authors declare that there are no conflicts of interest regarding the publication of this article.

\section{Acknowledgments}

This research is supported by NSFC (no. 71201023, no. 71371051, and no. 71671037), Social Science Fund Project of Jiangsu Province (no. 15GLC003), Humanities and Social Science Planning Foundation of the Ministry of Education of China (no. 16YJA630026), and Teaching and Research Program for Excellent Young Teachers of Southeast University (no. 2242015R30021).

\section{References}

[1] F. Allen and D. Gale, "Financial contagion," Journal of Political Economy, vol. 108, no. 1, pp. 1-33, 2000.

[2] E. Nier, J. Yang, T. Yorulmazer, and A. Alentorn, "Network models and financial stability," Journal of Economic Dynamics and Control, vol. 31, no. 6, pp. 2033-2060, 2007.

[3] R. M. May and N. Arinaminpathy, "Systemic risk: the dynamics of model banking systems," Journal of the Royal Society Interface, vol. 7, no. 46, pp. 823-838, 2010.

[4] P. Gai, A. Haldane, and S. Kapadia, "Complexity, concentration and contagion," Journal of Monetary Economics, vol. 58, no. 5, pp. 453-470, 2011.

[5] S. Li, "Contagion risk in an evolving network model of banking systems," Advances in Complex Systems. A Multidisciplinary Journal, vol. 14, no. 5, pp. 673-690, 2011.

[6] S. Li and J. He, "Fitness model for tiered structure in the interbank market," Complexity, vol. 17, no. 5, pp. 37-43, 2012.

[7] C.-P. Georg, "The effect of the interbank network structure on contagion and common shocks," Journal of Banking and Finance, vol. 37, no. 7, pp. 2216-2228, 2013.
[8] A. Sachs, "Completeness, interconnectedness and distribution of interbank exposures--a parameterized analysis of the stability of financial networks," Quantitative Finance, vol. 14, no. 9, pp. 1677-1692, 2014.

[9] A. Sensoy and B. M. Tabak, "Dynamic spanning trees in stock market networks: the case of Asia-Pacific", Physica A: Statistical Mechanics and its Applications, vol. 414, pp. 387-402, 2014.

[10] C. Aymanns and C.-P. Georg, "Contagious synchronization and endogenous network formation in financial networks," Journal of Banking and Finance, vol. 50, pp. 273-285, 2015.

[11] D. Acemoglu, A. Ozdaglar, and A. Tahbaz-Salehi, "Systemic risk and stability in financial networks," American Economic Review, vol. 105, no. 2, pp. 564-608, 2015.

[12] T. Chen, X. Li, and J. Wang, "Spatial interaction model of credit risk contagion in the CRT market," Computational Economics, vol. 46, no. 4, pp. 519-537, 2015.

[13] F. Betz, N. Hautsch, T. A. Peltonen, and M. Schienle, "Systemic risk spillovers in the European banking and sovereign network," Journal of Financial Stability, vol. 25, pp. 206-224, 2016.

[14] S. Li, X. Sui, and Q. Ma, "An endogenous network model of banking systems," Complexity, vol. 21, no. S1, pp. 512-520, 2016.

[15] S. Li and X. Sui, "Contagion risk in endogenous financial networks," Chaos, Solitons and Fractals, vol. 91, pp. 591-597, 2016.

[16] J. C. González-Avella, V. H. De Quadros, and J. R. Iglesias, "Network topology and interbank credit risk," Chaos, Solitons and Fractals, vol. 88, pp. 235-243, 2016.

[17] A. Sensoy, K. Ozturk, E. Hacihasanoglu, and B. M. Tabak, "Not all emerging markets are the same: a classification approach with correlation based networks," Journal of Financial Stability, 2015.

[18] T. Christiano Silva, M. Soares da Silva, and B. Miranda Tabak, "Financial networks and bank liquidity," The Journal of Network Theory in Finance, vol. 2, no. 4, pp. 1-51, 2016.

[19] T. C. Silva, S. R. S. de Souza, and B. M. Tabak, "Network structure analysis of the Brazilian interbank market," Emerging Markets Review, vol. 26, pp. 130-152, 2016.

[20] T. C. Silva, S. R. S. de Souza, and B. M. Tabak, "Structure and dynamics of the global financial network," Chaos, Solitons \& Fractals, vol. 88, pp. 218-234, 2016.

[21] T. C. Silva, S. M. Guerra, B. M. Tabak, and R. C. de Castro Miranda, "Financial networks, bank efficiency and risk-taking," Journal of Financial Stability, vol. 25, pp. 247-257, 2016.

[22] T. C. Silva, S. R. Souza, and B. M. Tabak, "Monitoring vulnerability and impact diffusion in financial networks," Journal of Economic Dynamics \& Control, vol. 76, pp. 109-135, 2017.

[23] L. Bargigli, G. di Iasio, L. Infante, F. Lillo, and F. Pierobon, "Interbank markets and multiplex networks: centrality measures and statistical null models," in Interconnected Networks, pp. 179-194, Springer International Publishing, New York, NY, USA, 2016.

[24] S. Boccaletti, G. Bianconi, R. Criado et al., "The structure and dynamics of multilayer networks," Physics Reports. A Review Section of Physics Letters, vol. 544, no. 1, pp. 1-122, 2014.

[25] G. F. de Arruda, E. Cozzo, Y. Moreno, and F. A. Rodrigues, "On degree-degree correlations in multilayer networks," Physica D. Nonlinear Phenomena, vol. 323/324, pp. 5-11, 2016.

[26] S. Poledna, J. L. Molina-Borboa, S. Martínez-Jaramillo, M. van der Leij, and S. Thurner, "The multi-layer network nature of systemic risk and its implications for the costs of financial crises," Journal of Financial Stability, vol. 20, pp. 70-81, 2015.

[27] A. Serguieva, "Multichannel contagion vs stabilisation in multiple interconnected financial markets," SSRN Electronic Journal, 2016, University College London. 
[28] C. León, J. Pérez, and L. Renneboog, "A multi-layer network of the sovereign securities market," Borradores de Economa 840, Banco de la República de Colombia, 2014.

[29] S. Langfield, Z. Liu, and T. Ota, "Mapping the UK interbank system," Journal of Banking and Finance, vol. 45, no. 1, pp. 288303, 2014.

[30] J. Molina-Borboa, S. Martinez-Jaramillo, F. López-Gallo, and M. v. Leij, "A multiplex network analysis of the Mexican banking system: link persistence, overlap and waiting times," The Journal of Network Theory in Finance, vol. 1, no. 1, pp. 99-138, 2015.

[31] L. Bargigli, G. di Iasio, L. Infante, F. Lillo, and F. Pierobon, "The multiplex structure of interbank networks," Quantitative Finance, vol. 15, no. 4, pp. 673-691, 2015.

[32] I. Aldasoro and I. Alves, "Multiplex interbank networks and systemic importance: an application to European data," Journal of Financial Stability, 2016.

[33] N. Musmeci, V. Nicosia, T. Aste et al., "The multiplex dependency structure of financial markets," 2016, https://arxiv.org/abs/ 1606.04872 .

[34] M. E. Newman, "Mixing patterns in networks," Physical Review E: Statistical, Nonlinear, and Soft Matter Physics, vol. 67, no. 2, 2003.

[35] P. Bonacich, "Factoring and weighting approaches to status scores and clique identification," Journal of Mathematical Sociology, vol. 2, pp. 113-120, 1972.

[36] S. P. Borgatti, "Centrality and network flow," Social Networks, vol. 27, no. 1, pp. 55-71, 2005. 


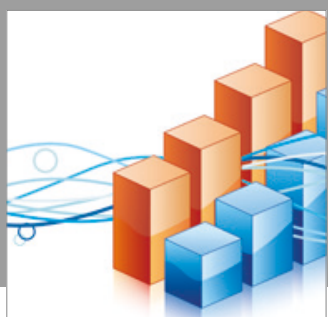

Advances in

Operations Research

vatersals

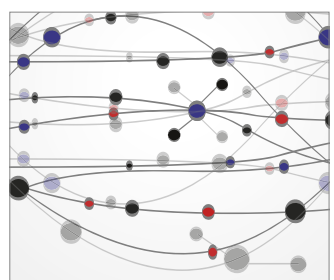

\section{The Scientific} World Journal
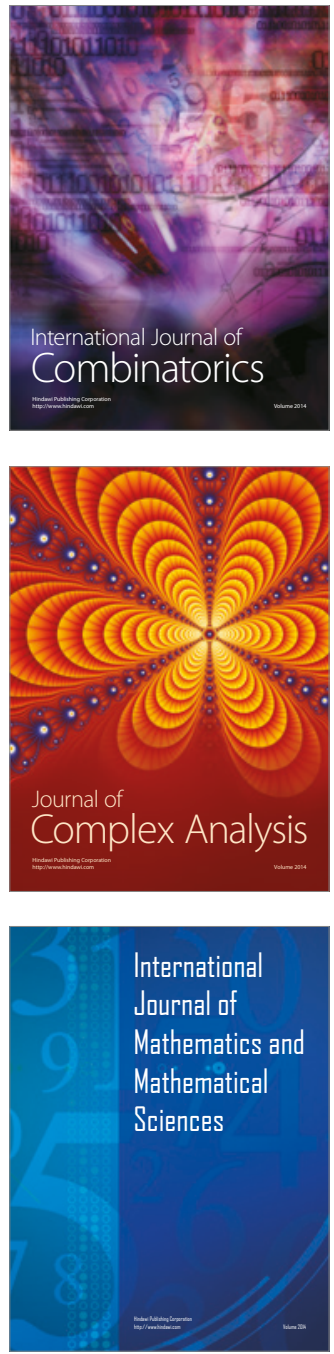
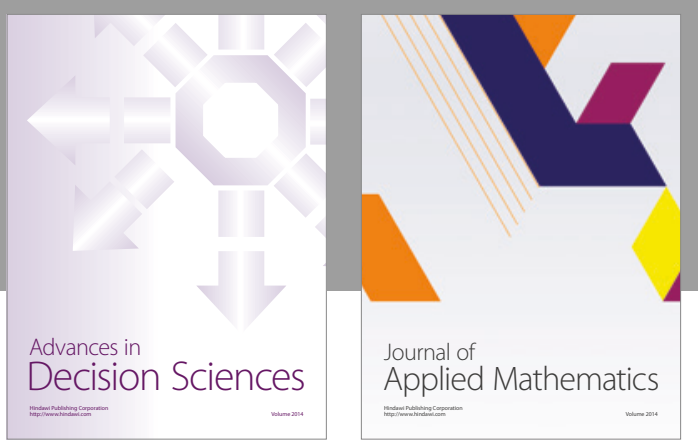

Algebra

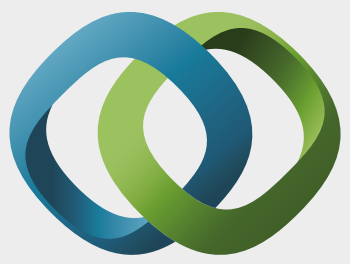

\section{Hindawi}

Submit your manuscripts at

https://www.hindawi.com
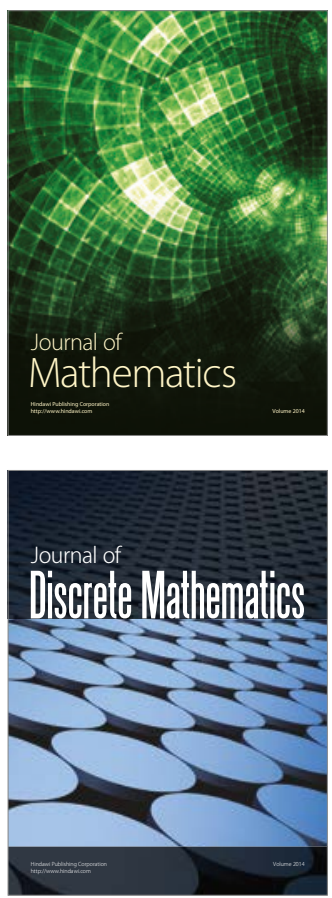

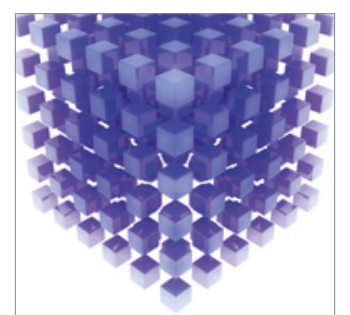

Mathematical Problems in Engineering
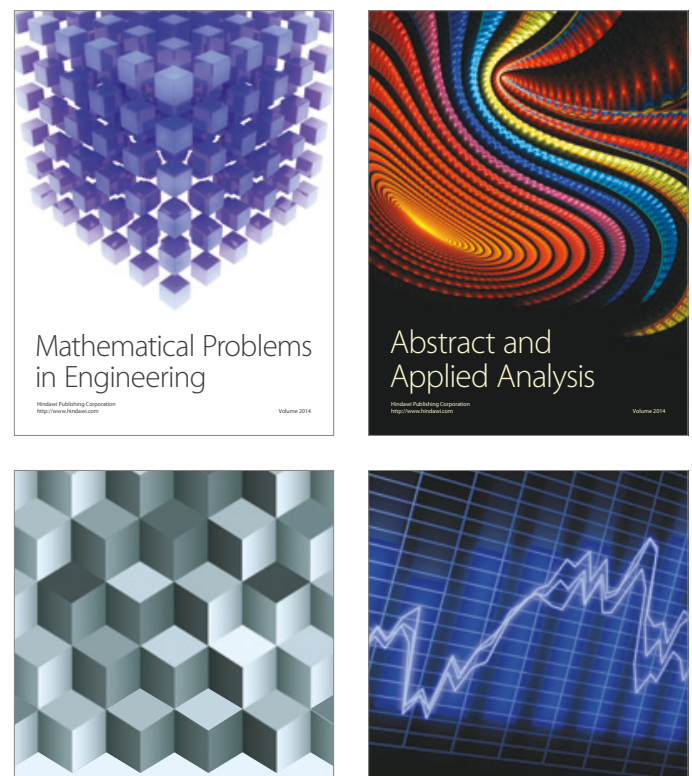

Journal of

Function Spaces

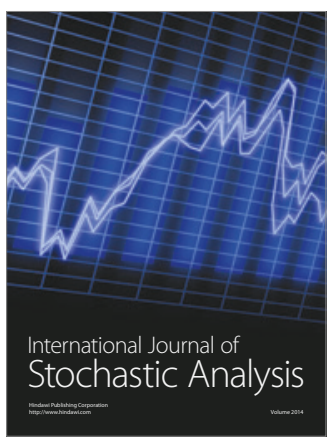

Probability and Statistics
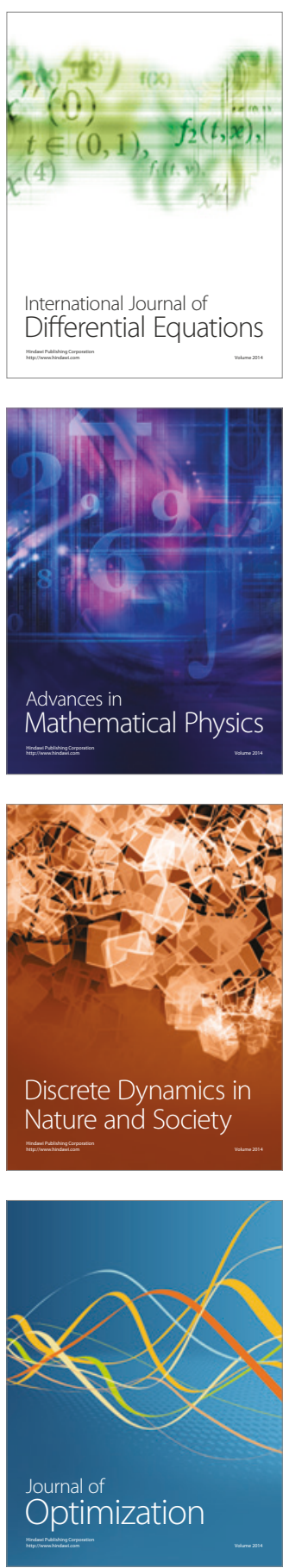